\title{
Effect of a semirigid thoracolumbar orthosis on gait and sagittal alignment in patients with an osteoporotic vertebral compression fracture
}

This article was published in the following Dove Medical Press journal:

Clinical Interventions in Aging

\author{
Eva Jacobs' \\ Rachel Senden ${ }^{2}$ \\ Christopher McCrum ${ }^{2,3}$ \\ Lodewijk W van Rhijn' \\ Kenneth Meijer $^{2}$ \\ Paul CWillems' \\ 'Department of Orthopaedic Surgery, \\ Research School CAPHRI, Maastricht \\ University Medical Center+, \\ Maastricht 6229 HX, the Netherlands; \\ ${ }^{2}$ Department of Nutrition and \\ Movement Sciences, NUTRIM \\ School of Nutrition and Translational \\ Research in Metabolism, Maastricht \\ University Medical Center+, \\ Maastricht, the Netherlands; ${ }^{3}$ Institute \\ of Movement and Sport Gerontology, \\ German Sport University Cologne, \\ Cologne, Germany
}

Correspondence: Eva Jacobs Department of Orthopaedic Surgery, Research School CAPHRI, Maastricht University Medical Center+, 25 P Debyelaan, Maastricht $6229 \mathrm{HX}$,

the Netherlands

$\mathrm{Tel}+3 \mid 433884152$

Fax +3I 433874893

Email e.jacobs@maastrichtuniversity.nl
Background: An important goal in the treatment of osteoporotic vertebral compression fractures (OVCFs) is the prevention of new vertebral fractures and the subsequent progression to global sagittal malalignment. Current conservative treatment is multimodal and comprises analgesics, medication for osteoporosis, and physical therapy. However, little is known about the value of orthoses in the treatment of OVCFs.

Aims: The primary purpose of this study was to examine the direct effect of a semirigid thoracolumbar orthosis on gait in patients suffering from an OVCF. The secondary purpose was to evaluate changes in gait, radiographic sagittal alignment, pain, and quality of life over time. Methods: Fifteen postmenopausal patients with an OVCF were treated with a semirigid thoracolumbar orthosis. At baseline, after 6 weeks, and after 6 months, gait analysis was performed with a dual belt-instrumented treadmill with a $180^{\circ}$ projection screen providing a virtual environment (computer-assisted rehabilitation environment) combined with clinical and radiographic assessments.

Results: At baseline, bracing caused a significantly more upright posture during walking and patients walked faster, with larger strides, longer stride times, and lower cadence compared to walking without orthosis. After 6 weeks, radiographic and dynamic sagittal alignment had improved compared to baseline. The observed effect was gone after 6 months, when the orthosis was not worn anymore.

Conclusion: A semirigid thoracolumbar orthosis seems to have a positive effect on gait and stability in patients suffering from an OVCF, as was shown by a more upright posture, which may result in decreased compressive loading of the vertebrae. For studying the true effectiveness of dynamic bracing in the treatment of OVCFs, a prospective, randomized controlled trial will be needed.

Keywords: osteoporotic vertebral compression fracture (OVCF), orthosis, dynamic bracing, sagittal alignment, trunk motion, gait analysis, computer assisted rehabilitation environment

\section{Introduction}

Osteoporosis is characterized by low bone-mineral density and a resulting propensity for fractures, of which vertebral compression fractures are the most common clinical manifestation. In our aging society, the impact of osteoporotic vertebral compression fractures (OVCFs) on medical and economic costs is rapidly increasing. ${ }^{1}$

Postmenopausal women who present with an initial vertebral compression fracture (VCF) are at substantial risk of a subsequent vertebral fracture within 1 year. ${ }^{2}$ When fractured, the vertebra is commonly deformed by disproportionate height loss from the anterior vertebral body, resulting in wedging. ${ }^{3}$ Wedge accumulation over 
multiple thoracolumbar levels may lead to subsequent spinal deformity. ${ }^{4}$ Inability to compensate for the alternating load distribution of the spine leads to global spinal sagittal malalignment. ${ }^{4}$ Increased anterior spinal loading has been associated with a downward spiral of subsequent VCFs. ${ }^{5}$

In thoracolumbar hyperkyphosis, there is an increased anterior bending moment on the trunk, which shifts the body's center of mass forward, ie, closer to the anterior boundary of the base of support under the feet. It has been shown that global sagittal malalignment causes impaired postural control, slower gait, and a wider base of support with stance and gait. ${ }^{3,6}$ Impaired postural control during walking is a major risk factor for falls. ${ }^{7}$ Critically, falls among older adults who are frail and have osteoporosis are associated with high morbidity and mortality and may need high-cost medical interventions. ${ }^{1,8}$

Treatment of OVCFs should aim to break the downward spiral of recurrent VCFs and to prevent the subsequent progression of hyperkyphosis and resultant global sagittal malalignment. Furthermore, it should intend to prevent or slow the decline in postural control, while limiting the increasing risk of falling in these patients. ${ }^{7,9}$ Current conservative management of symptomatic OVCFs is multimodal and comprises analgesics, medication for osteoporosis, physical therapy, and bracing. ${ }^{10,11}$ The use of conventional, rigid spinal orthoses is limited in patients suffering from osteoporosis, due to suspected subsequent atrophy of the trunk muscles and restricted respiration, leading to low compliance. ${ }^{12,13}$ In order to address these drawbacks, the concept of "dynamic bracing" has been introduced, using semirigid thoracolumbar orthoses in which trunk-muscle strength is improved and compliance increased. ${ }^{12-15}$ These orthoses are commonly used to treat traumatic vertebral fractures, but there is little knowledge on their use for OVCFs. ${ }^{10,11,13,16}$ The primary goal of dynamic bracing in the conservative treatment of OVCFs is to reduce pain by stabilizing the spine and allowing for muscle-spasm relief. ${ }^{13}$ Another goal is to maintain neutral spinal alignment and to limit flexion, thus reducing anterior axial loading on fractured vertebrae. ${ }^{14}$ However, the actual effect of dynamic bracing on the sagittal alignment of the spine in patients suffering from an OVCF has only been determined by quantification of the regional kyphosis angle on static radiography. ${ }^{12,13}$ No studies are available evaluating the effect of a dynamic orthosis on gait and global sagittal alignment (GSA) of the spine. ${ }^{12,14,15}$ Data in previous studies regarding balance has mainly been retrieved during static tasks, such as standing, but it is important to note that stability control during static tasks shows little relationship with more dynamic tasks performed during activities of daily living. ${ }^{7,8,17}$
The primary aim of this study was to examine the direct effect of a semirigid thoracolumbar orthosis in patients suffering from an OVCF on spatiotemporal gait parameters, trunk motion, and stance-phase knee flexion while walking. The secondary study purpose was to evaluate changes over time in gait, radiographic sagittal alignment of the spine, and pain and quality of life in these patients.

\section{Methods \\ Participants}

This was an observational, single-center study approved by the Maastricht University Medical Centre institutional review board (NL52978.057.15). The study was explained before obtaining patients' written informed consent and conducted in accordance with the Declaration of Helsinki. Inclusion of participants was conducted from September 2015 to March 2018. Fifteen consecutive adult postmenopausal patients presenting at the emergency department suffering from a symptomatic new OVCF of the thoracolumbar spine were enrolled. Inclusion criteria were female sex, age 55 years or older, symptomatic OVCF ( $<3$ weeks old, visible on thoracolumbar radiography), and ambulatory. Exclusion criteria were unstable fractures, previous OVCFs, not fully ambulatory, need for walking aids, active cancer, and body weight exceeding the equipment rating for the treadmill $(135 \mathrm{~kg})$. Demographic data included patient age, body height, body weight, and Charlson Comorbidity Index. Informed consent was obtained from all individual participants included in the study. Data were obtained at baseline (T0), 6 weeks (T1), and 6 months (T2) after baseline. Since this study was set up as an exploratory study, no power calculation was performed for the desired primary outcome measure.

\section{Treatment}

All subjects were prescribed to wear the thoracolumbar orthosis Osteolind Plus (Werkmeister, Wanfried, Germany) in the first 6 weeks for the entire day and optionally during the night. This orthosis is semirigid and consists of a malleable metal frame with soft padding and a system of belts with fleece. The orthoses were adjusted for each patient by an orthopedic technician. After the 6-week visit, patients were requested to wear the orthosis at least 6 hours daily, and after 3 months for at least 3 hours daily until the final follow-up visit at 6 months. The actual time patients wore the orthosis per day was reported in a patient diary during the entire study period. The diary was also used to monitor comfort and complications. Patients were referred to the 
rheumatology department for treatment of osteoporosis. Analgesic medication was prescribed, whereas physical therapy was not.

\section{Gait and stability analysis}

Gait measurements were performed in a computer-assisted rehabilitation environment (CAREN; Motekforce Link, Amsterdam, the Netherlands) by two certified clinical operators. The CAREN system includes a dual-belt treadmill with two instrumented force plates $(1,000 \mathrm{~Hz})$, a 12-camera motion-capture system (100 Hz; Vicon Motion Systems, Oxford, UK), and a virtual environment that provides optic flow on a $180^{\circ}$ semicylindrical screen. Participants wore a safety harness connected to an overhead frame. Ten retroreflective markers were attached to anatomical landmarks (two acromion markers, four pelvic markers, two lateral distal femur markers, and two lateral malleolus markers) and were tracked by the motion-capture system. ${ }^{18}$ Marker tracks were filtered using a low-pass second-order Butterworth filter (zero-phase) with a cutoff frequency of $12 \mathrm{~Hz}$. Foot touchdown and "toe-off" were determined using the treadmill force plates ( $50 \mathrm{~N}$ threshold) in combination with a markerbased method. ${ }^{12}$ This combined method was used to be able to account accurately for foot touchdowns and toe-offs occurring in the centre of the treadmill triggering both force plates simultaneously. For all steps, the foot-marker method was used and then corrected based on the average discrepancy between the force-plate method and marker-method timing for all steps that contacted only one force plate.

Participants first completed a familiarization trial consisting of 90 seconds walking at $1 \mathrm{~m} /$ second followed by 90 seconds walking at self-paced speed. Following familiarization, patients walked under two conditions: firstly, without wearing the orthosis and subsequently while wearing the orthosis. During each condition, patients first walked at self-paced mode for 90 seconds and subsequently at a fixed speed of $1 \mathrm{~m} /$ second for 90 seconds.

Collected gait data were analyzed in MatLab version 9.4 using previously published algorithms. ${ }^{19,20}$ Spatiotemporal parameters calculated were: walking speed, defined as the average treadmill speed measured during self-paced mode; step frequency, defined as the inverse of the average duration between two subsequent heel strikes; step width, calculated as the mediolateral (ML) distance between the ankle markers at the moment of heel contact; and step length, defined as the anteroposterior (AP) distance between these markers at the moment of heel contact. Stability was expressed using margins of stability (MoS), calculated in both AP and ML directions at foot touchdown as the AP or
ML distance between the boundary of the base of support (the ankle marker) and the extrapolated center of mass in the corresponding plane, as defined by Hof et al. ${ }^{21}$ For estimation of center-of-mass position and velocity, the average positions of the four pelvis markers were used. Stance-phase knee flexion was determined as knee flexion measured during terminal stance. Trunk motion, defined as the position of the trunk with regard to the pelvis, was calculated as the AP distance between the average of both acromion markers and the middle of the pelvis markers. The average distance over a gait cycle was calculated. Positive values indicated forward positioning of the trunk (positive sagittal alignment), whereas negative values indicated backward leaning. Produced gait and stability parameters were based on all recorded steps. For analysis, the averages of all these parameters were used.

\section{Radiographic assessment}

Static sagittal alignment was analyzed on standardized lateral full-spine radiography using validated software (Surgimap; Nemaris, New York, NY, USA). Pelvic parameters measured were pelvic incidence, pelvic tilt, and sacral slope. Regional spinal parameters included lumbar lordosis (L1-S1) and thoracic kyphosis (T4-T12). Sagittal alignment was assessed by sagittal vertical axis, GSA, and global alignment and proportion (GAP) score. ${ }^{22,23}$ The wedge angle of each fractured vertebra was measured (the angle formed between the two lines drawn parallel to the superior and inferior end plates of the fractured vertebra). All patients had bone mineral-density measurements of lumbar spine using dual-energy X-ray absorptiometry. The WHO classification was used to define osteoporosis as $\mathrm{T}$-score $\leq-2.5$ (or osteopenia as $\mathrm{T}$-score $<1.0$ ) at the lumbar spine.

\section{Quality-of-life assessment}

Pain was assessed using a visual analogue scale of $0-10$, where 10 implies extreme pain and 0 no pain at all. Quality of life was assessed using the Quality of Life Questionnaire of the European Foundation for Osteoporosis (QUALEFFO 41).

\section{Statistics}

Data were analyzed using SPSS version 25 for Windows. To investigate the effect of bracing on gait, paired $t$-tests were performed. Linear mixed-effect model analysis (LSD correction) was performed to test for differences in gait, stability, trunk motion, radiographic sagittal alignment, pain, and quality of life among the various time points. A significance level of 0.05 was used. 


\section{Results}

Fifteen female participants with a symptomatic OVCF were included (mean age 69 [55-78] years, mean weight $69 \pm 11 \mathrm{~kg}$, mean height $1.61 \pm 0.06 \mathrm{~m}$, mean T-score $-1.65 \pm 1.41$ for the lumbar spine; Table 1). For the secondary purpose, 6-week and 6-month data were available for eleven patients, since four patients were not able or not willing to have follow-up measurements for various reasons. All patients wore the orthosis the entire day for the first 6 weeks; however, after the 6 -week visit, the time wearing the orthosis varied considerably among the participants (1-12 hours per day). Patients did not report any discomfort or complications wearing the orthosis.

\section{Effect of bracing}

At baseline, walking with the orthosis resulted in a significantly decreased trunk motion compared to walking without it (51\% decline, $P=0.006)$. In addition, while wearing the orthosis, patients tended to walk more quickly, with larger strides, longer stride times, and lower cadence (Table 2). Stance-phase knee flexion decreased insignificantly when walking with the orthosis; however, large variation existed among patients, as indicated by large SDs. Step width and MoS were comparable between both walking conditions (Table 2). Six weeks after the fracture, spatiotemporal parameters, MoS, stance-phase knee flexion, and trunk motion were comparable between the two walking conditions (Figure 1B). Six months after the fracture, there was again significantly reduced trunk motion when walking with the orthosis (Figure 1B versus C). The other outcome measures were comparable between the two walking conditions at 6 months after baseline.

\section{Changes over time}

\section{Gait and stability analysis (walking without orthosis)}

Patients showed a gradual improvement over time in trunk motion (decrease of $8 \%[P=0.66]$ and $21 \%[P=0.27]$ at 6 weeks and 6 months, respectively). Spatiotemporal parameters improved significantly compared to baseline, with faster walking (increase of $14 \%$ at 6 weeks $[P=0.004]$ and $19 \%$ at 6 months $[P<0.001])$, larger strides $(P=0.039$ at 6 weeks [4\%] and $P=0.007$ at 6 months $[5 \%])$, longer stride times $(P=0.008$ at 6 weeks $[5 \%]$ and $P=0.003$ at 6 months $[6 \%])$, and lower cadence $(P=0.011$ at 6 weeks $[4 \%]$ and $P=0.003$ at 6 months [4\%]; Table 3). Step width decreased over time, but the decrease with regard to baseline was significant only at 6 months $(P=0.041)$. Also, stability changed over time, showing decreased MoS in the ML direction and increased
$\mathrm{MoS}$ in the AP direction at 6 weeks $(P=0.077$ and $P=0.148$, respectively) and six months $(P=0.137$ and $P=0.045)$ with regard to baseline (Table 3 ). Stance-phase knee flexion was comparable among the time points, showing differences smaller than $2^{\circ}$. Changes in gait and stability between 6 weeks and 6 months were not significant.

\section{Radiographic assessment}

After 6 weeks of wearing the orthosis, patients demonstrated an improvement in static sagittal alignment with respect to baseline, determined by significantly decreased GSA (54.64 \pm 8.49 vs $48.31 \pm 7.64, P=0.001)$ and GAP score (5.64 \pm 2.87 vs $3.18 \pm 2.86, P=0.001)$, and decreased sagittal vertical axis $(50.05 \pm 39.53$ vs $33.65 \pm 36.37, P=0.089$; Figure $2 \mathrm{~A}-\mathrm{C})$, despite a significantly increased wedge angle (9.75 \pm 5.03 vs $13.69 \pm 4.79, P<0.001$; Figure 2D). However, after 6 months, when patients stopped wearing the orthosis, a rebound phenomenon was seen, as the GSA and GAP score

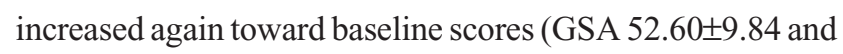

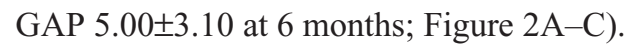

\section{Quality-of-life assessment}

With regard to baseline, patients reported less pain and improved quality of life 6 weeks after fracture, showing decreased pain on the visual analogue scale $(68 \%, P<0.001)$, QUALEFFO 41 pain $(38 \%, P<0.001)$, physical function (42\%,P=0.001), social function $(21 \%, P=0.091)$, and general health $(16 \%, P=0.138)$ scores. These reductions in pain and improvement in quality of life continued after the 6-week visit, showing significantly improved quality-of-life outcomes at 6 months compared to baseline. The improvements between 6 weeks and 6 months (range 6\%-40\%) were smaller than the improvements observed during the first 6 weeks (range 16\%-68\%; Figure 3).

\section{Discussion}

In the current study, the use of a semirigid thoracolumbar orthosis had a positive effect on dynamic sagittal balance in patients suffering an OVCF, as shown at baseline by a significant posterior shift in trunk motion when walking with the orthosis compared to walking without it (Table 2). Also, spatiotemporal parameters were positively affected by the orthosis in the majority of patients; however, because of variability, these observed changes were not significant at the group level (Table 3). After 6 weeks, static sagittal alignment (radiographs) and trunk motion during walking had improved compared to baseline pain, and quality of 


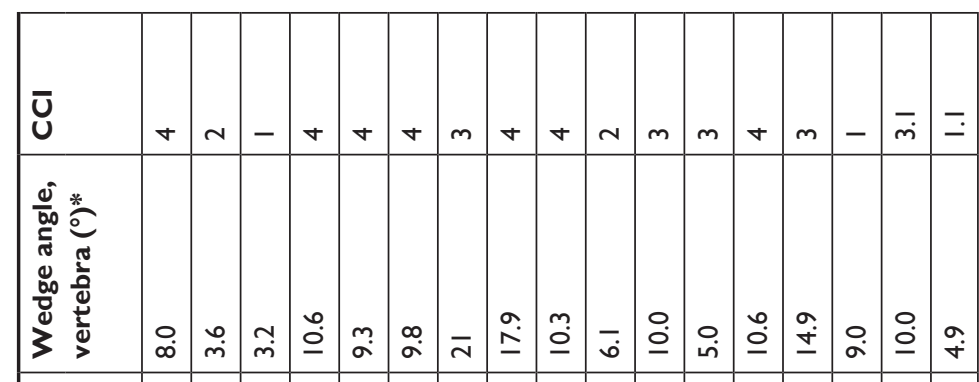

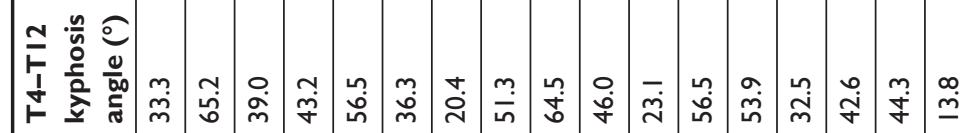

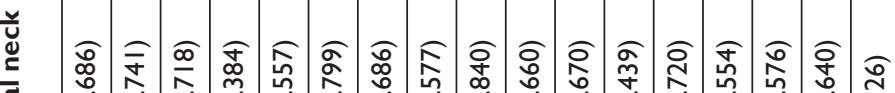

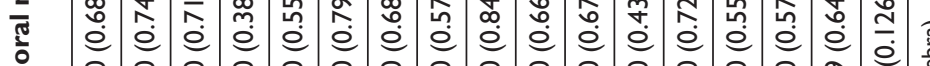

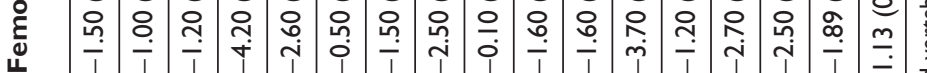
(1)

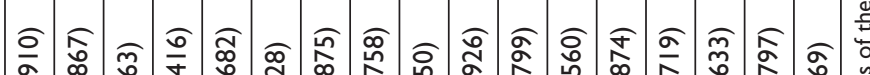

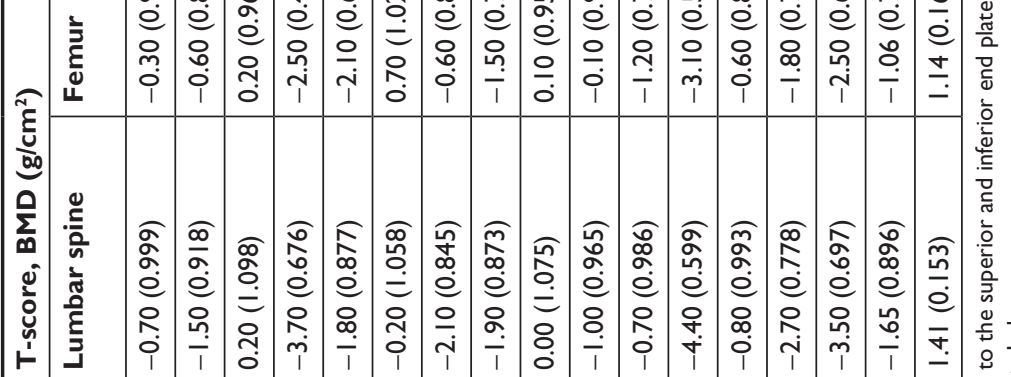

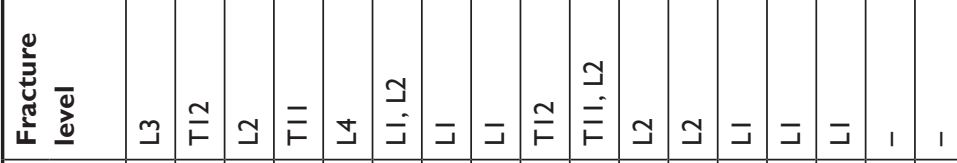

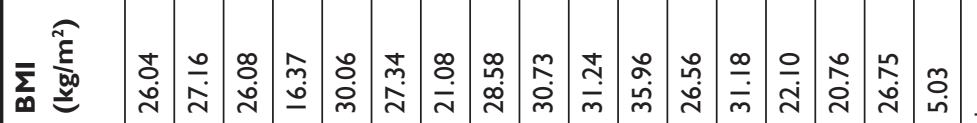

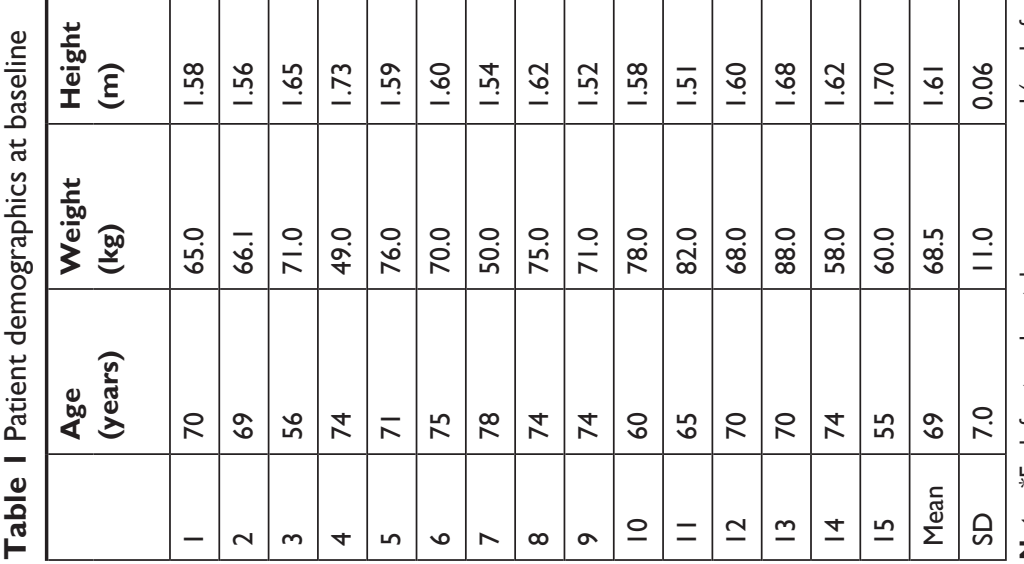

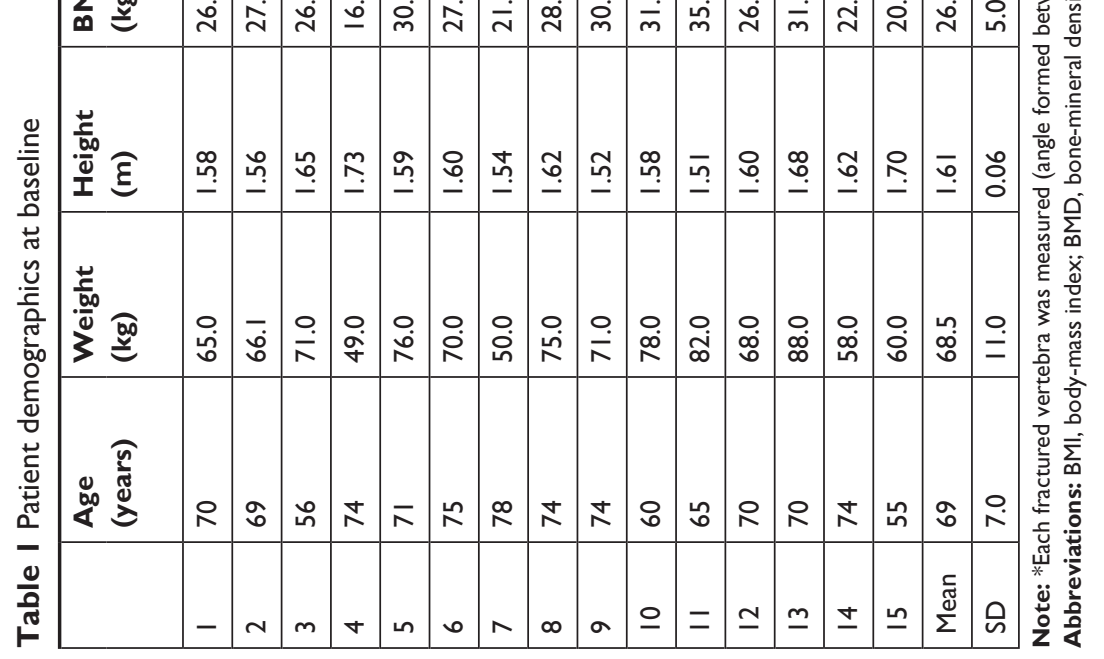


Table 2 Summary of the spatiotemporal and stability parameters, trunk motion, and stance phase knee flexion at baseline (mean + SD)

\begin{tabular}{|l|l|l|l|}
\hline Gait & $\begin{array}{l}\text { No brace } \\
(\mathbf{n}=\mathbf{I 5})\end{array}$ & $\begin{array}{l}\text { Brace } \\
(\mathbf{n}=\mathbf{I 5})\end{array}$ & -value \\
\hline Stride length $(\mathrm{m})$ & $1.1 \mathrm{I}+0.05$ & $\mathrm{I} .13+0.06$ & 0.417 \\
\hline Speed $(\mathrm{m} / \mathrm{s})$ & $1.07+0.12$ & $\mathrm{I} .13+0.17$ & 0.109 \\
\hline Stride time (seconds) & $1.1 \mathrm{I}+0.05$ & $\mathrm{I} .13+0.06$ & 0.140 \\
\hline Step width $(\mathrm{m})$ & $0.22+0.03$ & $0.22+0.03$ & 0.685 \\
\hline Cadence (steps/min) & $108.99+5.42$ & $106.78+5.26$ & 0.156 \\
\hline Trunk motion $(\mathrm{cm})$ & $3.60+2.59$ & $2.38+2.84$ & $0.006 *$ \\
\hline Knee flexion $\left({ }^{\circ}\right)$ & $7.37+6.87$ & $6.76+6.02$ & 0.305 \\
\hline Stability & \multicolumn{5}{|l}{} \\
\hline MoS ML $(\mathrm{m})$ & $0.05+0.01$ & $0.05+0.01$ & 0.675 \\
\hline MoS AP $(\mathrm{m})$ & $-0.08+0.03$ & $-0.07+0.03$ & 0.882 \\
\hline
\end{tabular}

Note: $* P<0.05$ between walking with and without orthosis.

Abbreviations: MoS, margins of stability; ML, mediolateral; AP, anteroposterior.

life improved significantly over the entire study period. Another interesting finding of this study was that it is possible to gain detailed information on gait and stability safely in a frail population with acute pain. A computer-assisted rehabilitation environment allows a safe and standardized condition to analyze gait and stability accurately in frail patients.

The results of this study suggest that 6 weeks of continuous bracing with a semirigid orthosis results in a more upright posture and adjusted gait pattern, comparable to walking with the orthosis. However, for improvement in posture and gait pattern and significant improvement in pain and quality of life, the natural healing process of the fractured vertebra might also play an important role. After 6 months, static and dynamic sagittal alignment deteriorated again. The deterioration in trunk motion may suggest that the improvement in sagittal alignment is slightly reversible. This could be attributed to the fact that patients did not wear the orthosis continuously any more after 6 weeks. In contrast, pain and quality of life had further improved after 6 weeks, suggesting that the changes in trunk motion are not caused solely by pain. This suggests that the continuous use of a semirigid orthosis might also contribute to improved sagittal alignment 6 weeks after baseline. ${ }^{10}$ Although the average absolute improvement in sagittal alignment was small, approximately $5^{\circ}$ in radiographic sagittal alignment
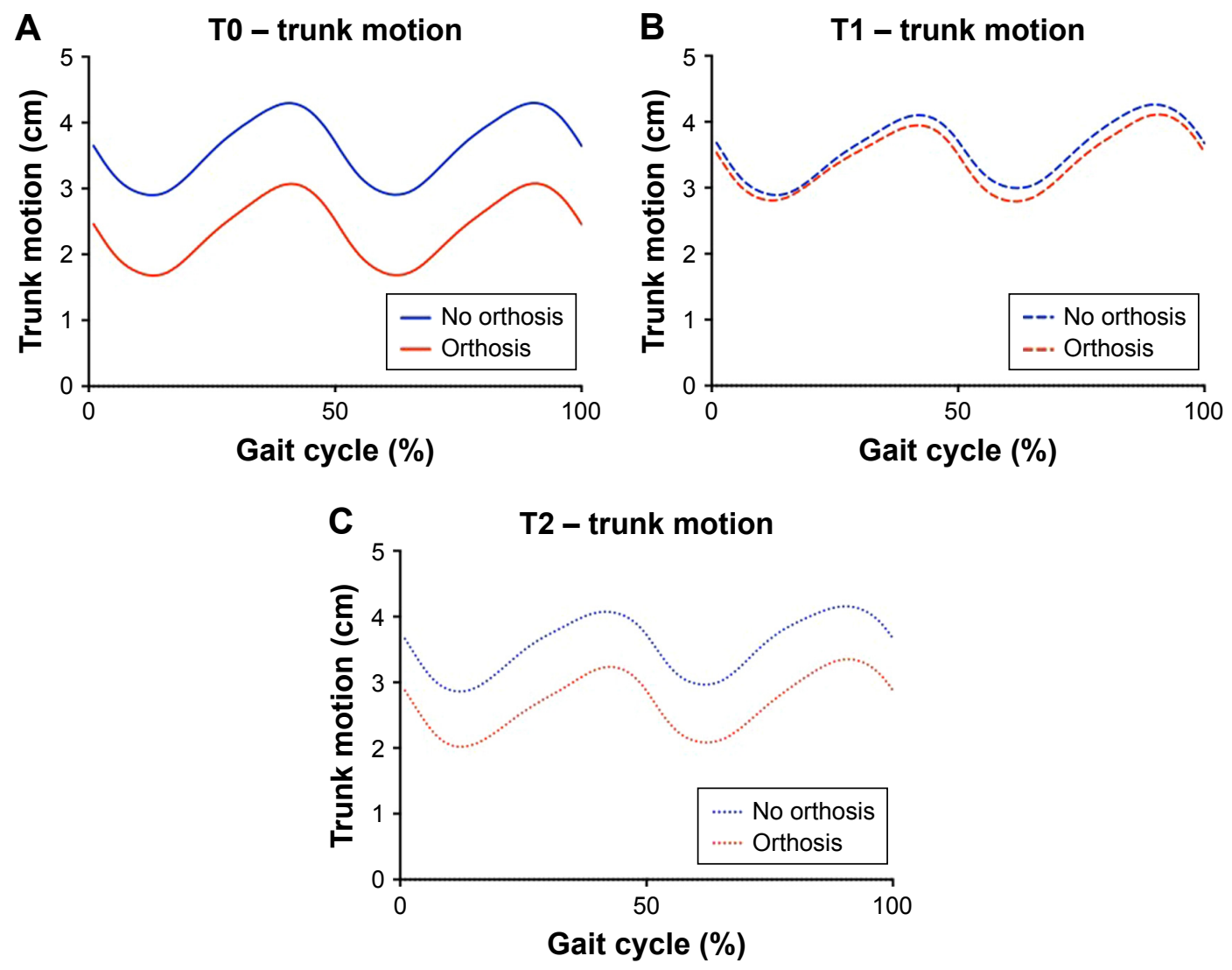

Figure I Trunk motion over time. Higher values indicate more anterior position of the trunk in comparison to the pelvis.

Notes: (A) Trunk motion at baseline. Significantly more flexed posture without orthosis $(P<0.05)$. (B) Trunk motion after 6 weeks wearing the orthosis showed no difference between walking with and without orthosis. (C) Trunk motion after 6 months, showing a pattern comparable to that found at baseline $(P<0.05)$. 
Table 3 Spatiotemporal and stability parameters, trunk motion, and stance phase knee flexion at the different time points (means \pm SD)

\begin{tabular}{|c|c|c|c|c|c|c|}
\hline Gait & TO $(n=I I)$ & TI $(n=I I)$ & T2 $(n=I I)$ & $\begin{array}{l}\text { T0 vs TI } \\
P \text {-value }\end{array}$ & $\begin{array}{l}\text { T0 vs T2 } \\
P \text {-value }\end{array}$ & $\begin{array}{l}\text { TI vs T2 } \\
P \text {-value }\end{array}$ \\
\hline Stride length (m) & $1.10 \pm 0.05$ & $1.14 \pm 0.07$ & $1.15 \pm 0.07$ & $0.039 *$ & $0.007^{*}$ & 0.414 \\
\hline Speed $(\mathrm{m} / \mathrm{s})$ & $1.09 \pm 0.10$ & $1.24 \pm 0.21$ & $1.30 \pm 0.23$ & $0.004^{*}$ & $<0.00 I^{*}$ & 0.252 \\
\hline Stride time (s) & $1.09 \pm 0.05$ & I. $14 \pm 0.07$ & $1.15 \pm 0.06$ & $0.008^{*}$ & $0.003^{*}$ & 0.613 \\
\hline Step width (m) & $0.22 \pm 0.03$ & $0.21 \pm 0.02$ & $0.20 \pm 0.03$ & 0.255 & $0.04 I^{*}$ & 0.321 \\
\hline Cadence (steps/min) & $110.67 \pm 5.06$ & $106.19 \pm 6.17$ & $105.05 \pm 5.70$ & $0.01 I^{*}$ & $0.003^{*}$ & 0.524 \\
\hline Trunk motion $(\mathrm{cm})$ & $3.60 \pm 2.59$ & $3.30 \pm 2.64$ & $2.85 \pm 2.79$ & 0.656 & 0.274 & 0.511 \\
\hline Knee flexion $\left({ }^{\circ}\right)$ & $6.27 \pm 7.55$ & $8.6 I \pm 4.84$ & $7.56 \pm 6.66$ & 0.126 & 0.367 & 0.545 \\
\hline \multicolumn{7}{|l|}{ Stability } \\
\hline MoS ML (m) & $0.05 \pm 0.01$ & $0.04 \pm 0.01$ & $0.04 \pm 0.01$ & 0.077 & 0.137 & 0.760 \\
\hline $\operatorname{MoS}$ AP $(m)$ & $-0.10 \pm 0.03$ & $-0.09 \pm 0.02$ & $-0.09 \pm 0.03$ & 0.148 & $0.045^{*}$ & 0.538 \\
\hline
\end{tabular}

Note: $* P<0.05$. T0, baseline; TI, 6 weeks after baseline; T2, 6 months after baseline.

Abbreviations: MoS, margins of stability; ML, mediolateral; AP, anteroposterior.

and $2 \mathrm{~cm}$ in trunk motion, it is considered clinically relevant. Bruno et $\mathrm{al}^{24}$ found that even a small increase in thoracic kyphosis results in a significant increase in vertebral compressive loading. In osteoporosis, the biomechanical strength of the vertebral bodies is reduced, especially in the anterior column. ${ }^{25}$ Subsequent compressive overload during loading in flexion causes progressive collapse of the unsupported anterior cortex, which damages the end plates and supporting trabeculae of the vertebrae, resulting in novel vertebral fractures. ${ }^{26}$ Wei et $\mathrm{al}^{27}$ found that VCFs are approximately five- to tenfold more prevalent in patients with moderate or severe kyphosis of the thoracolumbar spine than in patients
A

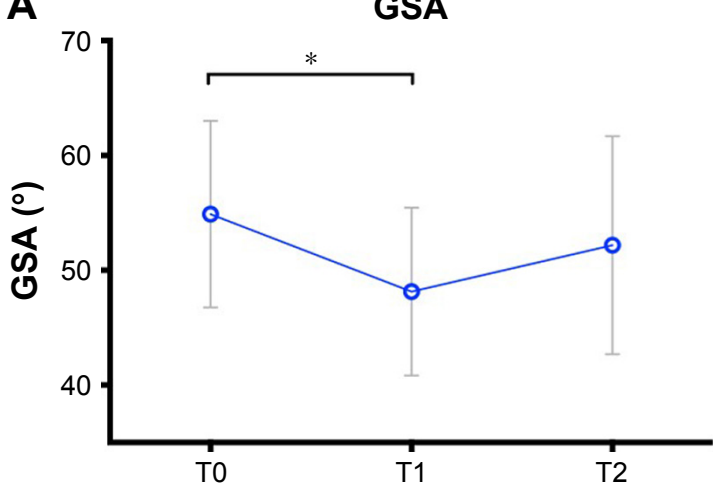

C

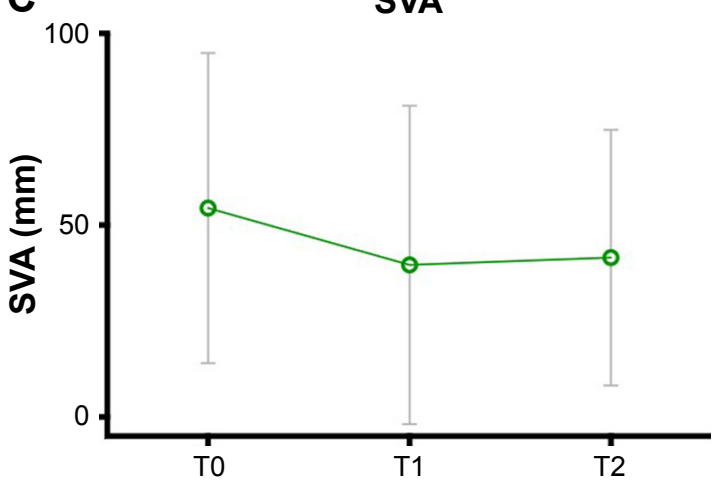

B

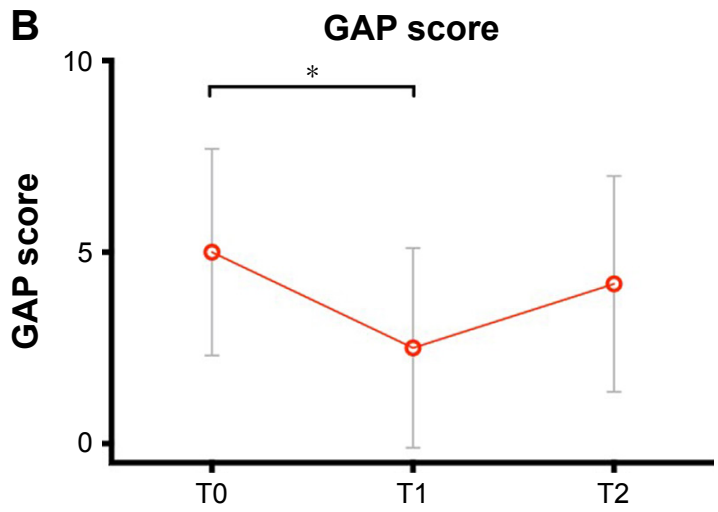

D

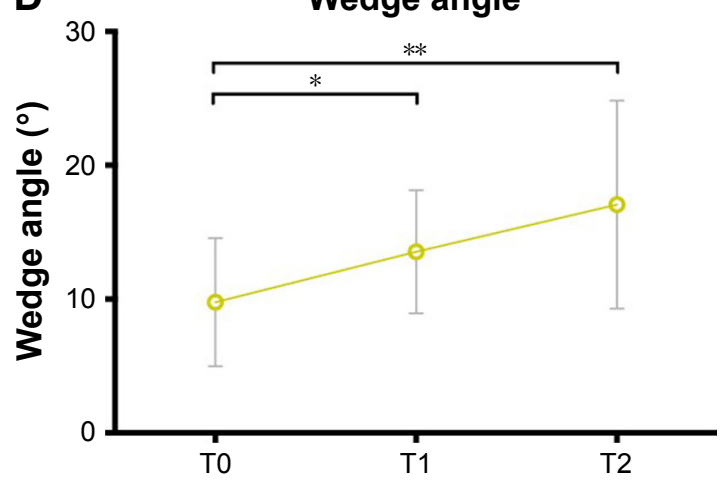

Figure 2 Radiographic sagittal balance. Graphs represent mean \pm SD at each time point.

Notes: $(A) G S A$, significant improvement T0 versus TI $(P<0.001)$, relapse at T2. (B) GAP score, significant improvement T0 versus TI ( $P=0.00 \mathrm{I})$, relapse at T2. (C) SVA, improvement T0 versus TI ( $P=0.089)$, relapse at T2. (D) Wedge angle, significant increase at 6 weeks $(* P<0.00 \mathrm{I})$ and 6 months $(* * P<0.00 \mathrm{I})$.

Abbreviations: GSA, global sagittal alignment; GAP, global alignment and proportion; SVA, sagittal vertical axis. 
A

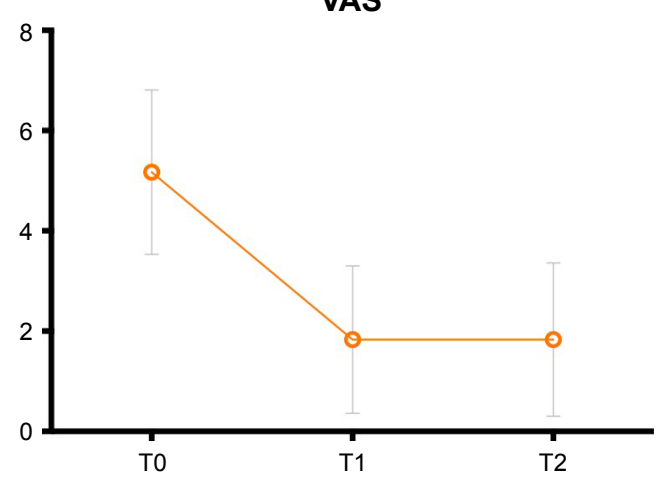

B

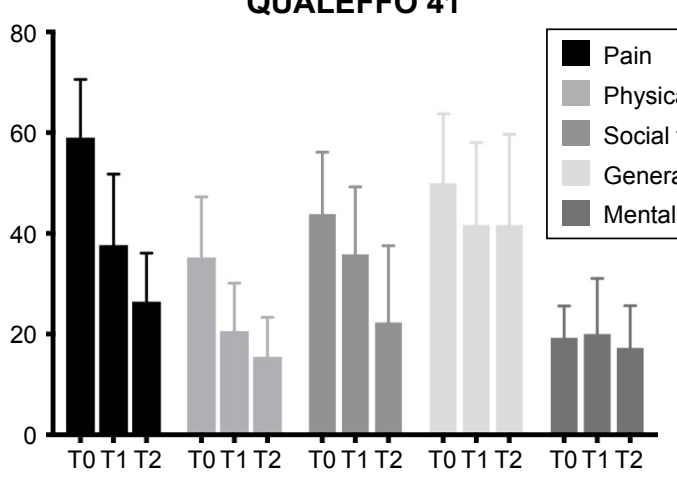

Figure 3 Pain and quality of life. Graphs represent mean \pm SD.

Notes: (A) Statistically significant improvement in VAS score over time (T0 vs TI $P<0.00$ I, T0 vs T2 P<0.00I). (B) QUALEFFO 4 I results. Statistically significant improvement over time in the QUALEFFO pain domain (T0 vs TI $P<0.00$ I, T0 vs T2 $P<0.00$ I), physical function (T0 vs TI $P=0.00$ I, T0 vs T2 $P<0.00$ I), and social function (T0 vs TI P=0.09I, T0 vs T2 $P<0.001$ )

Abbreviations: VAS, visual analogue scale; QUALEFFO, Quality of Life Questionnaire of the European Foundation for Osteoporosis.

with mild kyphosis. Therefore, in order to minimize increase in spinal loading and prevent new fractures, prevention of increased thoracolumbar kyphosis is key in patients suffering an OVCF. However, a randomized controlled trial with a larger patient group is required to investigate further the exact role of dynamic bracing on gait and posture in relation to the natural healing process of vertebrae.

The improvement in sagittal alignment 6 weeks after baseline found in the current study might be attributed to an increase in back-extensor strength related to the increased antagonistic muscular activity while wearing the orthosis. Pfeifer et a $1{ }^{12}$ revealed that wearing an orthosis significantly increases back-extensor strength (72\%) and the abdominal flexor strength (44\%). The increase in muscle strength was correlated with a decreased kyphosis angle and a more upright posture. ${ }^{12}$ Valentin et al ${ }^{15}$ demonstrated that dynamic bracing was associated with an increase in back-extensor strength of $50 \%$. This is consistent with the findings of Lantz et al, ${ }^{28}$ who showed that wearing an orthosis increased the electrical activity of back muscles. Subsequently, the reversibility of the observed positive effect on sagittal alignment in this study may have been caused by the decline in skeletal muscle strength when patients stopped wearing the orthosis. Without exercise after the age of 30 years, muscle mass declines at a rate of 3\%-8\% each decade, due to apoptosis, loss of motor-neuron function, and a reduction in calciumpumping activity. ${ }^{29}$ Therefore, maintaining optimal muscle mass and muscle function by means of active supervised exercise therapy in patients suffering an osteoporotic vertebral fracture may play an important role in the prevention of sagittal malalignment and subsequent fractures. Measuring the actual effect of dynamic bracing on back-extensor strength should be considered in future controlled studies.
According to the Global Spine Care Initiative, conservative management of acute pain and recovery of function in adults with OVCFs should include early mobilization, exercise, medication for osteoporosis, spinal orthosis for pain relief only, and calcitonin for analgesic-refractory pain. ${ }^{10}$ There are no recommendations concerning stability, gait, or posture yet. Although preliminary, the results of the current study might indicate that dynamic bracing using a thoracolumbar spinal orthosis may be a useful addition to the multimodal treatment of OVCFs, in order to maintain congruent posture. However, since the effect seemed to be reversible, lifetime lumbodorsal muscle exercises should be provided, in order to prevent subsequent vertebral fractures. ${ }^{9}$

This exploratory study was limited by a relatively small number of patients and did not incorporate a control group. Therefore, the results should be interpreted with caution. However, for the improvement in trunk motion and radiographic sagittal alignment, each patient served as their own control. Moreover, a study by Meccariello et al ${ }^{13}$ already revealed a significant reduction in pain and improvement in quality of life and respiratory function for patients treated with dynamic bracing. For studying the true effectiveness of dynamic bracing in the treatment of OVCFs, a large, prospective, randomized, controlled trial will be needed. In such a study, a subanalysis should be performed to evaluate the effect of fracture site and degree of compression on the main outcome parameters.

In conclusion, the results of the current study suggest a positive effect of a semirigid thoracolumbar orthosis on gait and stability in patients suffering an OVCF, as shown by a more upright posture, which may result in decreased compressive loading of the vertebrae. In addition, some evidence of a reversible effect on sagittal alignment was provided, suggesting the need for prolonged periods of bracing or 
lifetime lumbodorsal muscle exercises. However, the results need to be interpreted with caution, as natural recovery following OVCF may play a role. Further research is thus required, preferably by means of a randomized controlled trial on a larger patient group to confirm these results.

\section{Acknowledgments}

The authors thank Liesbeth Jütten and Mieke Vandewall, Department of Orthopaedic Surgery, Paul Willems, Department of Nutrition and Movement Science, and Rik Marcellis, Department of Physical Therapy for their valuable contribution to the execution of this study.

\section{Disclosure}

For the execution of or materials supplied for this study, no funding was received. CM was funded by the Kootstra Talent Fellowship awarded by the Centre for Research Innovation, Support, and Policy (CRISP) and by the NUTRIM Graduate Programme, both of Maastricht University Medical Center+. The other authors report no conflicts of interest in this work.

\section{References}

1. European Prospective Osteoporosis Study; Felsenberg D, Silman AJ, et al. Incidence of vertebral fracture in Europe: results from the European Prospective Osteoporosis Study (EPOS). J Bone Miner Res. 2002;17(4):716-724. doi:10.1359/jbmr.2002.17.4.716

2. Lindsay R, Silverman SL, Cooper C, et al. Risk of new vertebral fracture in the year following a fracture. JAMA. 2001;285(3):320-323.

3. Katzman WB, Wanek L, Shepherd JA, Sellmeyer DE. Age-related hyperkyphosis: its causes, consequences, and management. J Orthop Sports Phys Ther. 2010;40(6):352-360. doi:10.2519/jospt.2010.3099

4. Ailon T, Shaffrey CI, Lenke LG, Harrop JS, Smith JS. Progressive spinal kyphosis in the aging population. Neurosurgery. 2015;77(Suppl 4): S164-S172. doi:10.1227/NEU.0000000000000944

5. Broy SB. The vertebral fracture cascade: etiology and clinical implications. J Clin Densitom. 2016;19(1):29-34. doi:10.1016/j.jocd.2015.08.007

6. de Groot MH, van der Jagt-Willems HC, van Campen JPCM, Lems WF, Beijnen JH, Lamoth CJC. A flexed posture in elderly patients is associated with impairments in postural control during walking. Gait Posture. 2014;39(2):767-772. doi:10.1016/j.gaitpost.2013.10.015

7. van der Jagt-Willems HC, de Groot MH, van Campen JP, Lamoth CJ, Lems WF. Associations between vertebral fractures, increased thoracic kyphosis, a flexed posture and falls in older adults: a prospective cohort study. BMC Geriatr. 2015;15:34. doi:10.1186/s12877-015-0018-z

8. Sinaki M, Brey RH, Hughes CA, Larson DR, Kaufman KR. Balance disorder and increased risk of falls in osteoporosis and kyphosis: significance of kyphotic posture and muscle strength. Osteoporos Int. 2005;16(8):1004-1010. doi:10.1007/s00198-004-1791-2

9. Sinaki M, Itoi E, Wahner HW, et al. Stronger back muscles reduce the incidence of vertebral fractures: a prospective 10 year follow-up of postmenopausal women. Bone. 2002;30(6):836-841.

10. Ameis A, Randhawa K, Yu H, et al. The Global Spine Care Initiative: a review of reviews and recommendations for the non-invasive management of acute osteoporotic vertebral compression fracture pain in low- and middle-income communities. Eur Spine J. 2017;27(Suppl 6): 861-869

11. Rzewuska M, Ferreira M, McLachlan AJ, Machado GC, Maher CG. The efficacy of conservative treatment of osteoporotic compression fractures on acute pain relief: a systematic review with meta-analysis. Eur Spine J. 2015;24(4):702-714. doi:10.1007/s00586-015-3821-5
12. Pfeifer M, Kohlwey L, Begerow B, Minne HW. Effects of two newly developed spinal orthoses on trunk muscle strength, posture, and quality-of-life in women with postmenopausal osteoporosis: a randomized trial. Am J Phys Med Rehabil. 2011;90(10):805-815. doi:10.1097/ PHM.0b013e31821f6df3

13. Meccariello L, Muzii VF, Falzarano G, et al. Dynamic corset versus three-point brace in the treatment of osteoporotic compression fractures of the thoracic and lumbar spine: a prospective, comparative study. Aging Clin Exp Res. 2017;29(3):443-449. doi:10.1007/s40520-016-0602-x

14. Azadinia F, Kamyab M, Behtash H, Maroufi N, Larijani B. The effects of two spinal orthoses on balance in elderly people with thoracic kyphosis. Prosthet Orthot Int. 2013;37(5):404 410. doi:10.1177/03093 64612474487

15. Valentin GH, Pedersen LN, Maribo T. Wearing an active spinal orthosis improves back extensor strength in women with osteoporotic vertebral fractures. Prosthet Orthot Int. 2014;38(3):232-238. doi:10.1177/ 0309364613497393

16. Kim HJ, Yi J-M, Cho H-G, et al. Comparative study of the treatment outcomes of osteoporotic compression fractures without neurologic injury using a rigid brace, a soft brace, and no brace: a prospective randomized controlled non-inferiority trial. J Bone Joint Surg Am. 2014; 96(23):1959-1966. doi:10.2106/JBJS.N.00187

17. McCrum C, Eysel-Gosepath K, Epro G, et al. Associations between bipedal stance stability and locomotor stability following a trip in unilateral vestibulopathy. J Appl Biomech. 2017;33(2):112-117. doi:10. 1123/jab.2016-0004

18. Hak L, Houdijk H, Steenbrink F, et al. Speeding up or slowing down?: gait adaptations to preserve gait stability in response to balance perturbations. Gait Posture. 2012;36(2):260-264. doi:10.1016/j.gaitpost. 2012.03.005

19. Hak L, Houdijk H, Steenbrink F, et al. Stepping strategies for regulating gait adaptability and stability. J Biomech. 2013;46(5):905-911. doi:10.1016/j.jbiomech.2012.12.017

20. Zeni JA Jr, Richards JG, Higginson JS. Two simple methods for determining gait events during treadmill and overground walking using kinematic data. Gait Posture. 2008;27(4):710-714. doi:10.1016/j.gaitpost. 2007.07.007

21. Hof AL, Gazendam MG, Sinke WE. The condition for dynamic stability. J Biomech. 2005;38(1):1-8. doi:10.1016/j.jbiomech.2004.03.025

22. Smith MW, Annis P, Lawrence BD, Daubs MD, Brodke DS. Acute proximal junctional failure in patients with preoperative sagittal imbalance. Spine J. 2015;15(10):2142-2148. doi:10.1016/j.spinee.2015.05.028

23. Yilgor C, Sogunmez N, Boissiere L, et al. Global Alignment and Proportion (GAP) Score: development and validation of a new method of analyzing spinopelvic alignment to predict mechanical complications after adult spinal deformity surgery. J Bone Joint Surg Am. 2017;99(19): 1661-1672. doi:10.2106/JBJS.16.01594

24. Bruno AG, Anderson DE, D'Agostino J, Bouxsein ML. The effect of thoracic kyphosis and sagittal plane alignment on vertebral compressive loading. J Bone Miner Res. 2012;27(10):2144-2151. doi:10.1002/ jbmr. 1658

25. Hou Y, Luo Z. A study on the structural properties of the lumbar endplate: histological structure, the effect of bone density, and spinal level Spine (Phila Pa 1976). 2009;34(12):E427-E433. doi:10.1097/BRS 0b013e3181a2ea0a

26. Landham PR, Gilbert SJ, Baker-Rand HLA, et al. Pathogenesis of vertebral anterior wedge deformity: a 2-stage process? Spine (Phila Pa 1976). 2015;40(12):902-908. doi:10.1097/BRS.0000000000000905

27. Wei Y, Tian W, Zhang GL, Lv YW, Cui GY. Thoracolumbar kyphosis is associated with compressive vertebral fracture in postmenopausal women. Osteoporos Int. 2017;28:1925-1929. doi:10.1007/s00198017-3971-x

28. Lantz SA, Schultz AB. Lumbar spine orthosis wearing. II. Effect on trunk muscle myoelectric activity. Spine (Phila Pa 1976). 1986;11(8): 838-842.

29. Flack KD, Davy KP, Hulver MW, Winett RA, Frisard MI, Davy BM. Aging, resistance training, and diabetes prevention. J Aging Res. 2010; 201:127315. 


\section{Publish your work in this journal}

Clinical Interventions in Aging is an international, peer-reviewed journal focusing on evidence-based reports on the value or lack thereof of treatments intended to prevent or delay the onset of maladaptive correlates of aging in human beings. This journal is indexed on PubMed Central, MedLine,

CAS, Scopus and the Elsevier Bibliographic databases. The manuscript management system is completely online and includes a very quick and fair peer-review system, which is all easy to use. Visit http://www.dovepress. com/testimonials.php to read real quotes from published authors.

Submit your manuscript here: http://www.dovepress.com/clinical-interventions-in-aging-journal 